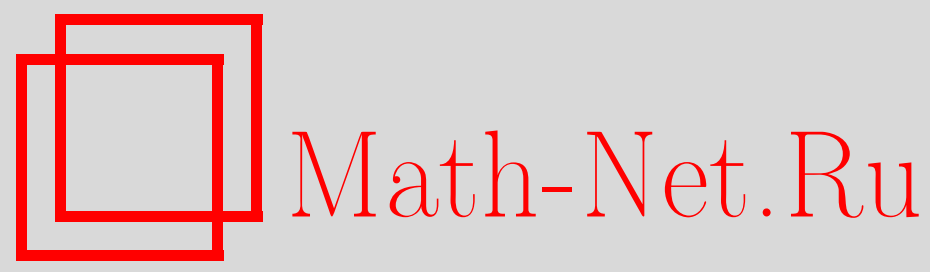

3. И. Бежаева, В. И. Оселедец, Об одной случайной динамической системе, Матем. заметки, 1997, том 61, выпуск 6, 803-809

DOI: https://doi.org/10.4213/mzm1564

Использование Общероссийского математического портала Math-Net.Ru подразумевает, что вы прочитали и согласны с пользовательским соглашением http://www.mathnet.ru/rus/agreement

Параметры загрузки:

IP: 54.198 .67 .100

26 апреля 2023 г., 13:45:10

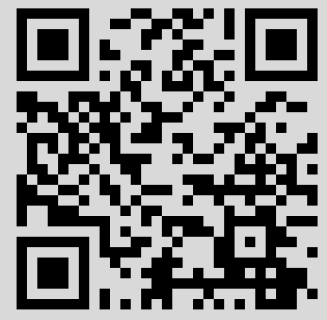




\section{ОБ ОДНОЙ СЛУЧАЙНОЙ ДИНАМИЧЕСКОЙ СИСТЕМЕ}

З.И. Бежаева, В.И. Оселедец

Рассматривается дискретная динамическая система

$$
X_{n+1}=2 \sigma \cos \left(2 \pi \theta_{n}\right) g\left(X_{n}\right), \quad n \in \mathbb{Z},
$$

где $\theta_{n}$ - эргодический стационарный процесс, одномерное распределение которого равномерно на отрезке $[0,1], g(x)$ - нечетная непрерьвная ограниченная возрастающая функция, $\mathbb{Z}-$ кольцо целых чисел.

Доказьвается, что при некоторых условиях существует единственный стационарный процесс, являющийся решением указанных уравнений, и этот процесс имеет непрерывный чисто сингулярный спектр.

Библиографияя: 6 названий.

Введение. Рассмотрим дискретную динамическую систему

$$
X_{n+1}=2 \sigma \cos \left(2 \pi \theta_{n}\right) g\left(X_{n}\right), \quad n \in \mathbb{Z},
$$

где $\theta_{n}$ - эргодический стационарный процесс, одномерное распределение которого равномерно на отрезке $[0,1], g(x)$ - нечетная непрерывная ограниченная возрастающая функция, $\mathbb{Z}$ - кольцо целых чисел.

В некоторых физических работах (см. [1]) численно и экспериментально исследовались свойства случайного процесса $X_{n}$. При этом рассматривался случай $\theta_{n+1}=$ $\left\{\theta_{n}+\alpha\right\}$, где $\alpha \in(0,1)$ - иррациональное число, фигурные скобки означают дробную часть числа, $g(x)=\tanh x$.

Результаты численного счета [1] показали, что при $\sigma=1.5, \alpha=(\sqrt{5}-1) / 2$ должен существовать единственный стационарный процесс $X_{n}$, являющийся решением (1), для которого $P\left(X_{n}=0\right)=0$. При этом процесс $X_{n}$ должен иметь непрерывный чисто сингулярньй спектр. Позднее было показано, что это явление естественно возникает в некоторых автономных динамических системах с непрерьвным временем [2].

Чтобы прояснить природу рассматриваемого явления, в данной работе будем изучать стационарньй процесс $\left(X_{n}, \theta_{n}\right)$, удовлетворяющий уравнениям $(1)$, в двух случаях:

$$
\text { 1) } g(x)=\left\{\begin{array}{cc}
-1, \quad x<-1, \\
x, \quad|x| \leqslant 1, \\
1, \quad x>1 ;
\end{array} \quad \text { 2) } g(x)=\left\{\begin{array}{cl}
\frac{x}{1-x}, & x<0 \\
\frac{x}{1+x}, & x \geqslant 0 .
\end{array}\right.\right.
$$

Работа выполнена при частичной поддержке Российского фонда фундаментальных исследований, грант № 96-01-00377, и Международного научного фонда, грант DSM-9300752. 
В п. 1 мы покажем, что если $\theta_{n}$ - эргодический стационарный процесс, одномерное распределение которого равномерно на отрезке $[0,1]$, и

$$
\int_{0}^{1} \ln (2 \sigma|\cos (2 \pi \theta)|) d \theta=\ln \sigma>0
$$

т.е. если $\sigma>1$, то для случаев 1) и 2) существует единственный стационарньй процесс $X_{n}$, удовлетворяющий уравнениям (1). При этом $\left(X_{n}, \theta_{n}\right)$ - стационарньй процесс и $P\left(X_{n}=0\right)=0$.

В п. 2 мы покажем, что процесс $X_{n}$ имеет непрерывньй чисто сингулярньй спектр, если $\theta_{n+1}=\left\{\theta_{n}+\alpha\right\}$, где $\alpha$ - иррациональное число.

1. Рассмотрим последовательность $\left(X_{n}, \theta_{n}\right)$, которая удовлетворяет рекуррентным соотношениям

$$
X_{n+1}=2 \sigma\left|\cos \left(2 \pi \theta_{n}\right)\right| g\left(X_{n}\right), \quad X_{n} \geqslant 0, \quad n \in \mathbb{Z},
$$

где $\left(\theta_{n}, n \in \mathbb{Z}\right)$ - эргодический стационарньй процесс, одномерное распределение которого равномерно на отрезке $[0,1]$. Как было сказано, мы будем рассматривать два случая:

$$
\text { 1) } g(x)=\left\{\begin{array}{ll}
x, & 0 \leqslant x \leqslant 1, \\
1, & x>1 ;
\end{array} \text { 2) } g(x)= \begin{cases}\frac{x}{1-x}, & x<0 \\
\frac{x}{1+x}, & x \geqslant 0\end{cases}\right.
$$

Нас будет интересовать стационарный процесс $X_{n}$, удовлетворяюший соотношенияМ (2).

Очевидно, что если $X_{n} \equiv 0$ для любого $n$, то $X_{n}$ является решением уравнения (2). Докажем следующее утверждение.

ТЕОремА. Если $\sigma>1$, то существует единственный стационарный прочесс $\left(X_{n}, \theta_{n}\right)$, удовлетворяющий уравнениям $(2)$, такой, что $P\left(X_{n}=0\right)=0$.

ДокАЗАТЕЛЬСТво. Введем обозначения

$$
\begin{gathered}
\sigma_{k}=\sigma\left(\theta_{-k}\right)=2 \sigma\left|\cos \left(2 \pi\left(\theta_{-k}\right)\right)\right|, \quad k=0,1,2, \ldots, \\
f_{\theta_{-k}}(x)=\sigma_{k} g(x), \quad f_{n, \omega}(x)=f_{\theta_{-1}}\left(f_{\theta-2 \alpha}\left(\cdots f_{\theta-n \alpha}(x)\right)\right), \quad n \geqslant 1,
\end{gathered}
$$

где $\omega$ - реализация процесса $\left(\theta_{n}, n \in \mathbb{Z}\right)$.

Из уравнений (1) следует, что $X_{0}=f_{n, \omega}\left(X_{-n}\right)$. Для доказательства теоремы потребуется следующая лемма.

ЛЕмма. При каждом $x>0$ при почти всех $\omega$ существует

$$
\lim _{n \rightarrow \infty} f_{n, \omega}(x)=\varphi(\theta),
$$

не зависящий от $x$. 
ДоКАЗАТЕЛЬСТВо леммы состоит из двух частей.

1) Сначала рассмотрим случай, когда

$$
g(x)= \begin{cases}x, & 0 \leqslant x \leqslant 1 \\ 1, & x>1\end{cases}
$$

Из определения $f_{\theta_{-k}}(x)$ и вида функции $g(x)$ можно вьвести, что

$$
f_{n, \omega}(x)= \begin{cases}\sigma_{n, \omega} x, & 0 \leqslant x \leqslant \frac{\tilde{\sigma}_{n}\left(\sigma_{1}, \ldots, \sigma_{n}\right)}{\sigma_{n, \omega}} \\ \tilde{\sigma}_{n}\left(\sigma_{1}, \ldots, \sigma_{n}\right), & x>\frac{\tilde{\sigma}_{n}\left(\sigma_{1}, \ldots, \sigma_{n}\right)}{\sigma_{n, \omega}}\end{cases}
$$

где

$$
\sigma_{n, \omega}=\prod_{i=1}^{n} \sigma_{i}=\prod_{i=1}^{n} \sigma\left(\theta_{-i}\right), \quad \tilde{\sigma}_{n}\left(\sigma_{1}, \ldots, \sigma_{n}\right) \leqslant \sigma_{1}, \quad n \geqslant 1 .
$$

По теореме Биркгофа для почти всех $\omega$ существует

$\lim _{n \rightarrow \infty} \frac{1}{n} \ln \sigma_{n, \omega}=\lim _{n \rightarrow \infty} \frac{1}{n} \sum_{k=1}^{n} \ln \sigma\left(\theta_{-k}\right)=E \ln \sigma_{i}=\int_{0}^{1} \ln (2 \sigma|\cos (2 \pi \theta)|) d \theta=\ln \sigma>0$.

Поэтому для почти всех $\omega$ существуют $n(\omega), c(\omega)$ такие, что для $n>n(\omega)$

$$
\sigma_{n, \omega}>c(\omega) \sigma^{n / 2}
$$

Значит, для почти всех $\omega$

$$
\lim _{n \rightarrow \infty} \frac{\tilde{\sigma}_{n}\left(\sigma_{1}, \ldots, \sigma_{n}\right)}{\sigma_{n, \omega}}=0
$$

Из соотношения

$$
f_{n, \omega}(x)=f_{n-1, \omega}\left(f_{\theta_{-n}}(x)\right)
$$

следует, что $\tilde{\sigma}_{n}\left(\sigma_{1}, \ldots, \sigma_{n}\right)=f_{n-1, \omega}\left(\sigma_{n}\right)$. Кроме того, для почти всех $\omega$ найдется $n_{1}(\omega)$ такое, что при $n>n_{1}(\omega)$

$$
\sigma_{n}>\frac{\tilde{\sigma}_{n-1}\left(\sigma_{1}, \ldots, \sigma_{n-1}\right)}{\sigma_{n-1, \omega}},
$$

так как, начиная с некоторого $n_{1}(\omega)$, в силу (4)

$$
\prod_{i=1}^{n} \sigma_{i}>\sigma_{1}>\tilde{\sigma}_{n-1}\left(\sigma_{1}, \ldots, \sigma_{n-1}\right)
$$

Из (4)-(7), а также из вида (3) функции $f_{n-1, \omega}(x)$ следует, что, начиная с некоторого $n_{2}(\omega)$,

$$
\tilde{\sigma}_{n}\left(\sigma_{1}, \ldots, \sigma_{n}\right)=\tilde{\sigma}_{n-1}\left(\sigma_{1}, \ldots, \sigma_{n-1}\right) .
$$

Последнее равенство и (5) означают, что для почти всех $\omega$ при любом фиксированном $x>0$ существует

$$
\lim _{n \rightarrow \infty} f_{n, \omega}(x)=\varphi(\omega)
$$


и этот предел не зависит от $x$.

2) Пусть теперь

$$
g(x)=\frac{x}{x+1}, \quad x>0 .
$$

Тогда функция $f_{\theta_{-k}}(x)=\sigma_{k} g(x)$ задает дробно-линейное преобразование. Дробно-линейному преобразованию $f_{\theta_{-k}}(x)$ соответствует матрица

$$
A_{\theta_{-k}}=\left(\begin{array}{cc}
\sigma_{k} & 0 \\
1 & 1
\end{array}\right)
$$

Преобразование $f_{n, \omega}(x)$ также является дробно-линейным и ему соответствует матрица

$$
A_{n, \omega}=A_{\theta_{-1}} \cdot A_{\theta_{-2}} \cdots A_{\theta_{-n}}=\left(\begin{array}{cc}
\sigma_{n, \omega} & 0 \\
c_{n, \omega} & 1
\end{array}\right) .
$$

Здесь, как и раньше,

$$
\sigma_{n, \omega}=\prod_{k=1}^{n} \sigma_{k}
$$

Из очевидного соотношения $A_{n+1, \omega}=A_{\theta_{-1}} A_{n, S^{-1} \omega}$, где $S$ - оператор сдвига в пространстве траекторий процесса $\theta_{n}$, следует, что $c_{n+1, \omega}=\sigma_{n, S^{-1} \omega}+c_{n, S^{-1} \omega}$.

Обозначим

$$
\frac{c_{n+1, \omega}}{\sigma_{n+1, \omega}}=\tilde{c}_{n+1, \omega}
$$

Тогда

$$
\tilde{c}_{n+1, \omega}=\frac{\sigma_{n, S^{-1} \omega}}{\sigma_{n+1, \omega}}+\frac{c_{n, S^{-1} \omega}}{\sigma_{n+1, \omega}}=\frac{1}{\sigma_{1}}+\frac{\tilde{c}_{n, S^{-1} \omega}}{\sigma_{1}} .
$$

Продолжая рекуррентно эту формулу, получаем, что при всех $\omega$

$$
\tilde{c}_{n+1, \omega}=\frac{1}{\sigma_{1}}+\frac{1}{\sigma_{1} \sigma_{2}}+\cdots+\frac{1}{\sigma_{1} \sigma_{2} \cdots \sigma_{n}} .
$$

Пусть

$$
c(\omega)=\sum_{n=1}^{\infty} \frac{1}{\prod_{k=1}^{n} \sigma_{k}} .
$$

Теперь из (4) следует, что при почти всех $\omega$ существует

$$
\lim _{n \rightarrow \infty} \tilde{c}_{n+1, \omega}=c(\omega)
$$

Отсюда, вновь используя (4), получаем, что при почти всех $\omega$ при любом $x>0$

$$
\lim _{n \rightarrow \infty} f_{n+1, \omega}(x)=\lim _{n \rightarrow \infty} \frac{\sigma_{n+1, \omega} x}{c_{n+1, \omega} x+1}=\lim _{n \rightarrow \infty} \frac{x}{\tilde{c}_{n+1, \omega} x+1 / \sigma_{n+1, \omega}}=\frac{1}{c(\omega)} .
$$

Лемма доказана.

Пусть теперь

$$
\varphi(\omega)=\lim _{n \rightarrow \infty} f_{n, \omega}(x)
$$


Очевидно, что для $\varphi(\omega)$ справедливо равенство $\varphi(S \omega)=f_{\theta_{0}}(\varphi(\omega))$. Легко видеть также, что процесс $\varphi(S \omega)=f_{\theta_{0}}\left(\varphi(\omega), \theta_{n}\right)$, где $\theta_{n}$ - равномерно распределенный на отрезке $[0,1]$ стационарньй процесс, удовлетворяющий уравнениям (2).

Покажем теперь, что этот стационарный процесс является единственным стационарным процессом, удовлетворяющим уравнениям (2), для которого $P\left(X_{n}=0\right)=0$.

Рассмотрим любой стационарньй процесс, удовлетворяющий уравнениям (2) и такой, что $P\left(X_{n}=0\right)=0$.

Пусть $P(\omega, \Delta)$ - условная вероятность того, что $X_{0} \in \Delta$ при условии, что фиксирована реализация процесса $\theta_{n}$.

Заметим, что при почти всех $\omega$ условное распределение $P(\omega, \Delta)$ сосредоточено в интервале $(0, \varphi(\omega)]$. Это следует из того, что при почти всех $\omega$ значение $X_{0}=f_{n, \omega}\left(X_{-n}\right)$ и, как было показано, при больших $n$ значение функции $f_{n, \omega}\left(X_{-n}\right)$ содержится в отрезке $(0, \varphi(\theta)]$.

Из стационарности $\left(X_{n}, \theta_{n}\right)$ и из $(2)$ следует, что

$$
P(\omega, \Delta)=P\left(S^{-n} \omega, f_{n, \omega}^{-1}(\Delta)\right) .
$$

Рассмотрим $\Delta \subseteq(0, \varphi(\omega))$. Пусть $f_{n, \omega}^{-1}(\Delta)=\Delta_{n}$. Тогда из (3) следует, что при больших $n$

$$
\Delta_{n} \subseteq\left(0, \frac{\tilde{\sigma}_{n}\left(\sigma_{1}, \ldots, \sigma_{n}\right)}{\sigma_{n, \omega}}\right)
$$

для случая 1$)$.

Для случая 2) имеем при больших $n$

$$
\Delta_{n} \subseteq\left(0, \exp \left(-n \ln \frac{\sigma}{2}\right)\right) .
$$

Рассмотрим теперь множество $A_{\varepsilon}=\{x: 0 \leqslant x<\varepsilon\}$. Из условия $P\left(X_{n}=0\right)=0$ получаем, что $P\left(A_{\varepsilon}\right)=\delta(\varepsilon) \rightarrow 0$ при $n \rightarrow \infty$.

Отсюда следует, что существует множество $B_{\varepsilon}$ такое, что для любого $\omega \in B_{\varepsilon}$ условная вероятность $P\left(\omega, A_{\varepsilon}\right)<\sqrt{\delta(\varepsilon)}$ и $P\left(B_{\varepsilon}\right)>1-\sqrt{\delta(\varepsilon)}$. Действительно, рассмотрим множество

$$
\bar{B}_{\varepsilon}=\left\{\omega: P\left(\omega, A_{\varepsilon}\right) \geqslant \sqrt{\delta(\varepsilon)}\right\} .
$$

Тогда

$$
P\left(\bar{B}_{\varepsilon}\right) \leqslant \frac{1}{\sqrt{\delta(\varepsilon)}} \int_{0}^{1} P\left(\theta, A_{\varepsilon}\right) d \theta=\sqrt{\delta(\varepsilon)} .
$$

Из (5) следует, что для некоторого $n(\omega, \varepsilon)$

$$
\frac{\tilde{\sigma}_{n}\left(\sigma_{1}, \ldots, \sigma_{n}\right)}{\sigma_{n, \theta} \sigma_{i}}<\varepsilon
$$

при всех $n>n(\theta)$. Поэтому для $n>n(\omega, \varepsilon)$ в случае 1$)$

$$
\Delta_{n}=f_{n, \omega}^{-1}(\delta) \subseteq[0, \varepsilon) .
$$

Для случая 2) имеем $\Delta_{n} \subseteq[0, \varepsilon)$, если $\exp (-n \ln (\sigma / 2))<\varepsilon$. 
Отсюда следует, что при достаточно больших $n$

$$
P\left(S^{-n} \omega, \Delta_{n}\right) \leqslant P\left(S^{-n} \omega, A_{\varepsilon}\right) .
$$

Известно [4], что существует строго возрастающая последовательность $\left\{n_{k}(\omega)\right\}$ такая, что $S^{-n_{k}(\omega)} \omega \in B_{\varepsilon}$. Отсюда следует, что при больших $k$

$$
P\left(S^{-n_{k}(\omega)} \omega, \Delta_{n_{k}(\omega)}\right) \leqslant P\left(S^{-n_{k}(\omega)} \omega, A_{\varepsilon}\right) \leqslant \sqrt{\delta(\varepsilon)} .
$$

Но $P(\theta, \Delta)=P\left(S^{-n_{k}(\omega)} \omega, \Delta_{n_{k}(\omega)}\right)$. Поэтому для любого $\varepsilon>0 P(\theta, \Delta) \leqslant \sqrt{\delta(\varepsilon)}$. Это означает, что $P(\theta, \Delta)=0$ для любых $\Delta \subseteq(0, \varphi(\omega))$. Следовательно, $P(\omega, \varphi(\omega))=1$.

Доказательство теоремы закончено.

2. В этом пункте мы докажем, что для рассматриваемых в данной работе функций $g(x)$ стационарный процесс $X_{n}\left(P\left(X_{n}=0\right)=0\right)$, удовлетворяющий уравнениям (1), имеет чисто непрерьвньй сингулярный спектр, если $\theta_{n+1}=\left\{\theta_{n}+\alpha\right\}$ и $\alpha$-любое иррациональное число.

Рассмотрим процесс $\left|X_{n}\right|$. Стационарньй процесс $\left|X_{n}\right|$ удовлетворяет уравнениям (2) и, как было показано, $P(\omega, \varphi(\omega))=1$. Но для рассматриваемого случая $\varphi(\omega)=\varphi_{0}\left(\theta_{0}\right)$, т.е. $\left|X_{n}\right|=\varphi_{0}\left(\theta_{n}\right)$.

Пусть $Y_{n}=\operatorname{sign} X_{n}$. Тогда $Y_{n}-$ стационарный процесс, удовлетворяющий уравнению

$$
\begin{gathered}
Y_{n+1}=\varepsilon\left(\theta_{n}\right) Y_{n}, \quad \theta_{n+1}=\left\{\theta_{n}+\alpha\right\}, \\
\varepsilon(\theta)=\operatorname{sign}(\cos (2 \pi \theta))=\left\{\begin{array}{c}
1, \quad 0<\theta<\frac{1}{4}, \frac{3}{4}<\theta<1, \\
-1, \quad \frac{1}{4}<\theta<\frac{3}{4} .
\end{array}\right.
\end{gathered}
$$

Следовательно,

$$
Y_{n}=\prod_{k=0}^{n-1} \varepsilon(\theta+k \alpha) Y_{0}
$$

Для стационарного процесса $X_{n}$ величина $Y_{n}$ принимает два значения $\{-1,1\}$.

Пусть $p(\theta, y)=P\left(Y_{0}=y \mid \theta_{0}=\theta\right)$. Введем динамическую систему $\left\{T^{n}\right\}$, где преобразование $T$ действует в пространстве пар $(\theta, y), y$ принимает два значения $\{-1,1\}$, по правилу

$$
T:(\theta, y) \mapsto(\theta+\alpha, \varepsilon(\theta) y) .
$$

Определим инвариантную меру $\mu$ как прямое произведение меры Лебега на $[0,1]$ на равномерную меру на двухточечном множестве $\{-1,1\}$.

Из определения функции $p(\theta, y)$ и стационарности процесса $Y_{n}$ следует, что

$$
p(\theta+\alpha, \varepsilon(\theta) y)=p(\theta, y)
$$

т.е. $p(\theta, y)-T$-инвариантная функция. Хорошо известно [3], [4], что $T$ - эргодическое преобразование, т.е. каждая $T$-инвариантная функция есть константа. Поэтому почти всюду $p(\theta, y)=1 / 2$, так как $\sum_{y} p(\theta, y)=1$. 
Процесс $Y_{n}$ можно считать функцией на пространстве пар $(\theta, y)$, а именно,

$$
Y_{n}=\prod_{k=0}^{n-1} \varepsilon(\theta+k \alpha) Y_{0}
$$

где $Y_{0}=Y_{0}(\theta, y)=y$. При таком задании процесса $Y_{n}=Y_{0}\left(T^{n}(\theta, y)\right)$.

Исходньй процесс $X_{n}$ может быть представлен в виде $X_{n}=X_{0}\left(T^{n}(\theta, y)\right)$, где $X_{0}(\theta, y)=\varphi_{0}(\theta) Y_{0}(\theta, y)$. Следовательно, спектральная мера процесса $X_{n}$ есть спектральная мера функции $X_{0}(\theta, y)$ относительно динамической системы $\left\{T^{n}\right\}$, так как

$$
E X_{n} X_{0}=\int X_{0}\left(T^{n}(\theta, y)\right) X_{0}(\theta, y) d \mu(\theta, y)
$$

Заметим, что пространство $H \subset L_{2}(\mu)$ функций вида $F(\theta) Y_{0}(\theta, y)$ инвариантно относительно динамической системы $\left\{T^{n}\right\}$. В частности,

$$
X_{0}=\varphi_{0}(\theta) Y_{0}(\theta, y) \in H,
$$

так как $\varphi_{0}(\theta)$ неотрицательная и ограниченная.

Оператор

$$
U: F(\theta, y) \mapsto F(T(\theta, y))
$$

динамической системы в пространстве $L_{2}(\mu)$ имеет в $H$ непрерьвньй спектр [3], [4].

Кроме того, известно, что в инвариантном пространстве $H$ спектр оператора $U$ сингулярен [5], [6]. Отсюда следует, что процесс $X_{n}$ имеет непрерывный чисто сингулярньй спектр.

Второй из авторов благодарит А. С. Пиковского, М. А. Закса, У. Фейдель, Ю. Куртца за полезные обсуждения.

Московский институт электроники и математики

Поступило

Московский государственный университет им. М.В. Ломоносова

04.05 .95

\section{СПИСОК ЦИТИРОВАННОЙ ЛИТЕРАТУРЫ}

[1] Pikovski A.S., Feudel U. Correlations and spectra of strange nonchaotic attractors // J. Phys. A. 1994. V. 27. P. 5209-5219.

[2] Pikovski A. S., Feudel U., Kurths J., Zaks M. A. Singular continious spectra in dissipative dynamics // Nichtlineare Dynamik. Preprint: Max-Planck-Arbeitsgruppe, 1994. P. 1-25.

[3] Оселедец В. И. О спектре эргодических автоморфизмов // Докл. АН СССР. 1968. Т. 168. № 5. C. 1009-1011.

[4] Корнфельд И. П., Синай Я. Г., Фомин С. П. Эргодическая теория. М.: Наука, 1980.

[5] Каток А. В., Стёпин А. М. Аппроксимации в эргодической теории // УМН. 1967. Т. 22. № 5. C. 81-106.

[6] Агеев О.Н. Перемешивание в компонентах перекладывания $T_{\alpha, \beta} / /$ УМН. 1994. Т. 49. №2. C. $143-144$. 